\title{
Semiclassical dynamics of quasi-one-dimensional, attractive Bose-Einstein condensates
}

\author{
Alexander Tovbis* \\ Department of Mathematics, University of Central Florida, Orlando, Florida 32816 \\ M. A. Hoefer \\ Department of Mathematics, North Carolina State University, Raleigh, NC 27695
}

\begin{abstract}
The strongly interacting regime for attractive Bose-Einstein condensates (BECs) tightly confined in an extended cylindrical trap is studied. For appropriately prepared, non-collapsing BECs, the ensuing dynamics are found to be governed by the one-dimensional focusing Nonlinear Schrödinger equation (NLS) in the semiclassical (small dispersion) regime. In spite of the modulational instability of this regime, some mathematically rigorous results on the strong asymptotics of the semiclassical limiting solutions were obtained recently. Using these results, "implosion-like" and "explosion-like" events are predicted whereby an initial hump focuses into a sharp spike which then expands into rapid oscillations. Seemingly related behavior has been observed in three-dimensional experiments and models, where a BEC with a sufficient number of atoms undergoes collapse. The dynamical regimes studied here, however, are not predicted to undergo collapse. Instead, distinct, ordered structures, appearing after the "implosion", yield interesting new observables that may be experimentally accessible.
\end{abstract}

PACS numbers: $03.75 . \mathrm{Kk}, 05.45 . \mathrm{Yv}$

\section{INTRODUCTION}

Bose-Einstein condensates (BECs) with attractive interactions between atoms have been found to exhibit collapse [1] resulting in violent, three-dimensional (3D) explosive dynamics $[2,3]$ and the propagation of quasione-dimensional (1D), stable bright solitary waves $[4,5]$. The implosion and subsequent explosion of a 3D BEC, a "Bosenova", has been explained theoretically as a blowup singularity of the Gross-Pitaevskii (GP) equation when the condensate has a sufficient number of atoms [6-8]. A quasi-1D soliton train was formed by exploiting this instability [4]. Other pattern forming instabilities in BECs include modulational and transverse instability [9] which result in the formation of coherent, localized nonlinear structures such as solitons and vortices [10]. In this work, we explore a new and completely different mechanism leading to violent dynamics and the formation of quasi-periodic nonlinear matter wave trains, the semiclassical (zero dispersion limit) regime where a quasi-1D attractive BEC can "implode" and "explode" yet does not undergo 3D collapse. This dynamical regime can be accessed with a cigar shaped BEC with negative s-wave scattering length of sufficiently small magnitude. Recent experiments suggest that this may be possible [11].

Exact, analytical results for the strong asymptotics of the small dispersion limit of the 1D focusing (attractive) Nonlinear Schrödinger equation (NLS) are used to describe the onset of a gradient catastrophe or sharp focusing of the condensate density (implosion) followed by breaking (explosion) $[12,13]$. We emphasize, however, that these dynamics do not correspond to collapse of the

${ }^{*}$ Electronic address: atovbis@pegasus.cc.ucf.edu
3D BEC wavefunction. Rather, the resulting dynamics reveal two counterpropagating radiative waves, the space between them filled by rapidly oscillating quasi-periodic (2-phase) nonlinear matter waves. Further dynamics are determined by the discrete spectrum (solitons) of the associated Zakharov-Shabat eigenvalue problem for the 1D focusing NLS. In the absence of the discrete spectrum, the pure radiation case, the amplitudes of the (2-phase) nonlinear matter waves are decaying exponentially in time [14]. In the presence of the discrete spectrum, (the number of points is inversely proportional to the semiclassical parameter) more complicated localized structures ( $2 n$-phase nonlinear waves) can appear within the oscillatory region with $n>1$ growing in time. The presence of an initial, sufficiently large inward BEC superfluid velocity (phase gradient) can completely remove the localized coherent structures in the oscillatory region [12].

In this work, we apply the aforementioned rigorous results to an attractive quasi-1D BEC in a cigar shaped trap. The small dispersion regime can be accessed by condensing a sufficiently large number of repulsive atoms in a properly sized cigar shaped trap. As has been done in the past $[2-5,11]$, a Feshbach resonance can then be applied to tune the sign of the nonlinear term, resulting in an attractive mean field interaction. While avoiding collapse of the entire condensate, the 1D implosion and explosion events are shown to be experimentally accessible for sufficiently tight radial confinement. Various observables resulting from the dynamics of the localized, prepared condensate are elucidated including the point of gradient catastrophe (breaking point), curves in the space-time plane, separating different asymptotic regimes (breaking curves), see Fig.1, and the asymptotic structure of the BEC density.

The outline of the paper is as follows. First, we in- 
troduce the appropriate parameter regime for the application of the semiclassical NLS equation to a BEC. Following this, we review recent rigorous results on the focusing NLS equation with small dispersion and discuss their application to single hump initial conditions.

\section{1D BEC AS A SEMICLASSICAL LIMIT OF THE FOCUSING NLS}

The temporal evolution of a BEC is governed by the GP equation for the condensate wave function $\Psi(\mathbf{r}, t)$, given by $[15,16]$

$$
i \hbar \frac{\partial}{\partial t} \Psi=\left[-\frac{\hbar^{2}}{2 m} \nabla^{2}+V(\mathbf{r}, t)+g(t)|\Psi|^{2}\right] \Psi,
$$

where: $m$ is the single atom mass, $V=V_{\perp}(y, z)+V_{\|}(x, t)$ is an external trapping potential with radial $V_{\perp}$ and axial $V_{\|}$terms, $g(t)=4 \pi \hbar^{2} a_{s}(t) / m$ is the nonlinear coefficient arising due to two-particle interactions and is characterized by the scattering length $a_{s}$. We will assume that

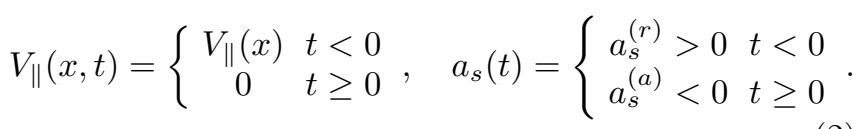

For $t<0$, the BEC is repulsive (positive scattering length) and confined in all three spatial dimensions. At $t=0$, the axial confinement is turned off and the scattering length is rapidly switched to a negative value, e.g. by a Feshbach resonance [15], so that the condensate becomes attractive.

We assume that the BEC has been prepared (for $t<0$ ) in the ground state of a strongly anisotropic trap

$$
V_{\perp}(y, z)=\frac{1}{2} m \omega_{r}^{2}\left(y^{2}+z^{2}\right),
$$

where $\omega_{r}$ is the harmonic trap frequency with radial localization width $a_{0}=\sqrt{\hbar / m \omega_{r}}$ The axial portion of the potential $V_{\|}(x)$, for $t<0$, confines the BEC's axial extent to a width $\Delta \gg a_{0}$.

Equation (1) conserves the particle number

$$
\int_{\mathbb{R}^{3}}|\Psi(\mathbf{r}, t)|^{2} d \mathbf{r}=N .
$$

A sufficient condition to avoid collapse in a harmonic potential is to take $N$ sufficiently small $N<N_{\text {cr }}$ [17]. In two spatial dimensions with a harmonic trap, it has been shown that $N_{\text {cr }}$ is related to the loss of stability of the nonlinear ground state $[7,18]$. Using stability theory for nonlinear ground states [19], one can compute an estimate of $N_{\mathrm{cr}}$. This result has been assumed to hold for 3D BECs as well, leading to numerical calculations of $N_{\text {cr }}$ for several trap configurations [8, 18, 20]. For the $3 \mathrm{D}$ harmonic potential $V(x, y, z)=V_{\perp}(y, z)$ that we are considering at $t \geq 0$, a numerical calculation in [20] determined

$$
N_{\mathrm{cr}} \approx 0.67 \frac{a_{0}}{\left|a_{s}^{(a)}\right|}
$$

We now consider the one-dimensional reduction of eq. (1) for $t>0$. This simplification requires that the characteristic energies of the radial excitations are much greater than the energies associated with the axial and nonlinear excitations $[21,22]$. This regime leads to the following requirement

$$
C \frac{N\left|a_{s}^{(a)}\right|}{\Delta} \ll 1
$$

where the $O(1)$ factor $C$ can be estimated through the initial density of the BEC (see further discussion in the Appendix). If (6) is satisfied, then $\Psi$ in eq. (1) for $t>0$ can be approximated by $[21,22]$

$$
\Psi(\xi, y, z, t) \approx \sqrt{\frac{N}{\Delta}} \phi(y, z) q(\xi / \Delta, t / k) e^{-i \omega_{r} t}
$$

where $\phi(y, z)=\exp \left[-\left(y^{2}+z^{2}\right) /\left(2 a_{0}^{2}\right)\right] /\left(\sqrt{\pi} a_{0}\right)$ is the $2 \mathrm{D}$, linear ground state for the transverse harmonic potential. Here, the axial variable $x$ has been replaced by $\xi$ for convenience. The remaining axial and temporal dependence is embodied in the function $q(x, \tau)$ which satisfies the NLS equation

$$
i \varepsilon q_{\tau}+\frac{\varepsilon^{2}}{2} q_{x x}+|q|^{2} q=0,
$$

where, with a slight abuse of notation, we re-introduce $x=\xi / \Delta$ which is now non-dimensional and set $\tau=t / k$. The parameters in eq. (8) are

$$
\varepsilon=\frac{a_{0}}{\left(2 N\left|a_{\mathrm{s}}^{(a)}\right| \Delta\right)^{1 / 2}}, \quad k=\Delta\left(\frac{m \Delta}{2\left|a_{s}^{(a)}\right| \omega_{r} N \hbar}\right)^{1 / 2} .
$$

We are interested in the semiclassical (small dispersion) regime where the semiclassical parameter $\varepsilon$ satisfies

$$
0<\varepsilon \ll 1 \text {. }
$$

Conservation of particle number in (4) combined with eq. (7) gives

$$
\int_{\mathbb{R}}|q(x, \tau)|^{2} d x=1 .
$$

In summary, we have derived the NLS equation (8) in the small dispersion regime with the assumptions of $N<$ $N_{\text {cr }}(5)$ and the inequalities (6) and (10). Experimentally, all of the parameters $a_{s}^{(a)}, N, \Delta$, and $a_{0}$ can be varied so that we expect that these three inequalities can be satisfied. By direct calculation, we find that all three inequalities (6), (10), and $N<N_{\text {cr }}$ can be satisfied by choosing, for example,

$$
N=\frac{a_{0}}{2\left|a_{s}^{(a)}\right|}, \quad \sqrt{\frac{a_{0}}{\Delta}} \ll 1, \quad \frac{C a_{0}}{2 \Delta} \ll 1 .
$$

The factor $C$ here (and in (6)) is chosen so that $\int_{\mathbb{R}}|q(x, \tau)|^{4} d x<C$. The inequalities in (12) are just the 
requirement of cigar shaped initial data with tight radial confinement. Using a Feshbach resonance, one can, in principle, tune the scattering length $a_{s}^{(a)}$ to an arbitrary value so that eq. (12) implies that any number of atoms is possible. In a recent experiment, the scattering length for ${ }^{7} \mathrm{Li}$ has been precisely tuned over seven orders of magnitude [11]. This demonstrates that the integrable, 1D NLS equation in the small dispersion limit may be a valid model for BEC experiments.

To illustrate the above discussion, consider the following data for the bright soliton experiments in [4] with ${ }^{7} \mathrm{Li}$. The parameters involved are $m \approx 10^{-26} \mathrm{~kg}$, $a_{s}^{(a)} \approx-1.6 \cdot 10^{-10} \mathrm{~m}, a_{0} \approx 1.6 \cdot 10^{-6} \mathrm{~m}$, and $N \approx 3 \cdot 10^{5}$. With these parameters, $N_{\mathrm{cr}} \approx 6700$ and the system is predicted to undergo collapse, as observed in the experiment. Nevertheless, assuming an initial axial width of $\Delta \approx 200 \mu \mathrm{m}$, which is the approximate experimental value, we calculate

$$
\varepsilon \approx 0.011, \quad k \approx 14 \mathrm{~ms}, \quad N\left|a_{s}^{(a)}\right| / \Delta \approx 0.24 .
$$

Equation (13) ensures the validity of the semiclassical regime for at least short times, whereas the quasi-1D assumption of eq. (13) is, perhaps, on the borderline of applicability.

\section{SUMMARY OF PREDICTIONS FROM RIGOROUS ASYMPTOTIC ANALYSIS}

Here we bring together and summarize some of the main results from the semiclassical, rigorous asymptotic analysis undertaken in $[12,14,23-27]$. Details can be found in Sections IV and V. Figure 1 from [28] depicts implosion and explosion dynamics generated by the single-hump initial condition $q(x, 0)=$ $e^{-x^{2}} \exp \left(-\frac{i}{\varepsilon} \ln \cosh x\right)$ for the NLS equation (8). Very similar dynamics were rigorously derived in [12] for the one parameter family of initial data

$$
q(x, 0, \varepsilon)=\operatorname{sech} x e^{-\frac{i \mu}{2 \varepsilon} \ln \cosh x},
$$

where $\mu \geq 0$ provides a measure of the phase gradient $(\arg q)_{x}$ or inward superfluid velocity in the context of BEC [15]. The key features of this study, illustrated by Figure 1, can be extended to generic decaying analytical one-hump initial data. here are some highlights:

- The physical $x, \tau$ plane is subdivided into regions where the solution is asymptotically (as $\varepsilon \rightarrow 0$ ) described by modulated $2 n$-phase nonlinear waves (the $n=0$ or plane wave approximation corresponds to the smooth region in Fig. $1, n=1$ to the next oscillatory region, etc.).

- Phase transitions between regions of different behavior are separated by breaking curves in the $x, \tau$ plane that do not depend on $\varepsilon$. Equations for the breaking curves are given by (26). A detailed description of the transitional behavior at the breaking curve can be found in [37];

- The tip of the breaking curve $\left(x_{0}, \tau_{0}\right)$ is the point of gradient catastrophe for the plane wave approximation. Behind this tip, the solution suddenly bursts into rapid amplitude oscillations or density spikes. Each spike within the vicinity of $\left(x_{0}, \tau_{0}\right)$ has the height $3\left|q\left(x_{0}, \tau_{0}\right)\right|$ and the shape of the rational breather solution to the NLS, while the locations of the spikes correspond to the poles of the special tritronquée solution to the first Painlevé equation, see [38]. For the family (14), the exact location of the point of gradient catastrophe is $\left(x_{0}, \tau_{0}\right)=\left(0, \frac{1}{\mu+2}\right)$ with $\left|q\left(x_{0}, \tau_{0}\right)\right|=\sqrt{\mu+2}$ so that the height of the spikes are $3 \sqrt{\mu+2}$; the slope of the breaking curve is $\frac{\cot \frac{\pi}{5}}{\sqrt{\mu+2}}$.

- The asymptotic solution for $q$ in the plane wave approximation region is completely characterized by the implicit formulas in eq. (19) when $\mu=2$ and eq. (20) when $\mu=0$. In the general case, it is determined by eq. (23).

- When the initial data (14) has $\mu \leq 2$, there exist $\mathcal{O}(1 / \varepsilon)$ points of discrete spectrum (solitons) centered at $x=0$. Their interaction leads to the secondary break starting at about $(x, \tau) \approx(0,1.5)$ in Fig. 1, followed by the region of 4-phase wave approximation.

- When the initial data (14) has $\mu>2$, there are no solitons. Therefore, no secondary breaking region exists and the solution contains only two regions of distinct behavior: smooth and oscillatory. The smooth region $(n=0)$ decays exponentially fast in time to zero whereas the background of the oscillatory region decays as $\mathcal{O}\left(\tau^{-1 / 2}\right)$ (however, the amplitude of the spikes decays exponentially to the background with $\tau$ ). A large inward, focusing momentum prevents the formation of higher breaking regions.

\section{SEMICLASSICAL LIMIT SOLUTIONS TO THE FOCUSING NLS}

In this subsection we discuss some mathematical background and recent analytical results related to the semiclassical regime of the focusing NLS. The focusing Nonlinear Schrödinger equation (8) is a universal, basic model for self-focusing and self-modulation in that it describes the evolution of the envelope of modulated waves in generic weakly nonlinear, dispersive systems. It is also one of the most celebrated nonlinear integrable equations that was first integrated by Zakharov and Shabat [29], who used the inverse scattering procedure to describe general decaying solutions $\left(\lim _{|x| \rightarrow \infty} q(x, 0)=0\right)$ 


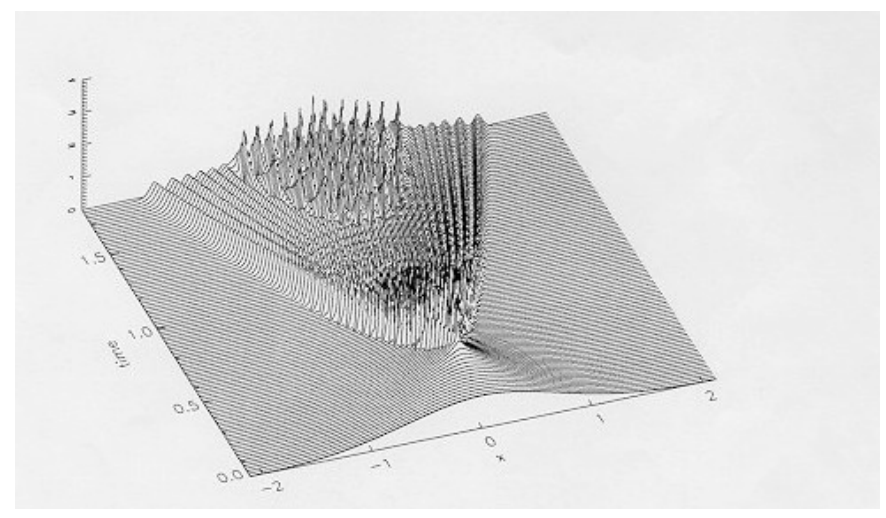

FIG. 1: Absolute value $|q(x, \tau, \varepsilon)|$ of a solution $q(x, \tau, \varepsilon)$ to the focusing NLS (8) versus $x, \tau$ coordinates. Here the initial data (15) is given by $A(x)=e^{-x^{2}}, S^{\prime}(x)=-\tanh x$, and $\varepsilon=0.02$. Reprinted from Handbook of Dynamical Systems, Vol 2, D. Cai, D. W. McLaughlin, and T. T. R. McLaughlin, The nonlinear Schrodinger equation as both a PDE and a dynamical system, pp 599-675, Copyright (2002) with permission from Elsevier.

in terms of radiation and solitons. Central to their discovery was the so-called Lax pair which effectively linearizes the NLS equation. The first equation in the Lax pair for the NLS is called the Zakharov-Shabat (ZS) system. It is used to define the correspondence between the initial data and the scattering data. Since the scattering data undergoes an explicit and very simple time evolution, the inverse scattering transform (IST), which maps the scattering data back to physical space, is used to obtain the evolution of given initial data at any time $\tau>0$.

In the semiclassical limit $(\varepsilon \rightarrow 0)$, the focusing NLS (8) exhibits modulationally unstable behavior (see Fig. 1), as was first shown in [30]. This is in drastic contrast to the case of the defocusing (repulsive) NLS equation $[28,31]$ in which the semiclassical theory shows regions of modulated periodic or quasi-periodic oscillation. These two very different types of behavior can be explained through the modulation equations, which are elliptic in the focusing case and hyperbolic in the defocusing case. The corresponding initial value problems are, therefore, illposed and well-posed respectively. As a result, a plane wave with amplitude modulated by $A(x)$ and phase modulated by $S(x)$, taken as initial data

$$
q(x, 0, \varepsilon)=A(x) e^{i S(x) / \varepsilon}
$$

for the focusing NLS (8), is expected to break immediately into disordered oscillations of both the amplitude and the phase. However, in the case of analytic initial data, the NLS evolution displays some orderly structure instead of the disorder suggested by the modulational instability, see [28, 32, 33]. Throughout this work, we will use the abbreviation NLS to mean "focusing Nonlinear Schrödinger equation".

Figure 1 from [28] depicts the time evolution of a typ- ical Gaussian-shaped, symmetric, analytic initial data (15). It identifies regions where different types of behavior (modulated $2 n$-phase nonlinear waves) of the solution $q(x, \tau, \varepsilon)$ appear. In particular, consecutive regions with $n=0,2$ and, presumably, $n=4$ are depicted in Fig. 1 . These regions are separated by curves in the $x, \tau$ plane that are called breaking curves or nonlinear caustics. The location of a breaking curve is defined by $A(x)$ and $S(x)$ from (15); it does not depend on $\varepsilon$. Within the $2 n$ phase wave approximation region, the strong asymptotics of $q(x, \tau, \varepsilon)$ can be expressed in terms of Riemann Thetafunctions (see, for example, [12]), that are defined on the genus $2 n$ hyperelliptic Riemann surface $\mathcal{R}(x, \tau)$. Therefore, the $2 n$-phase wave approximation region is referred to as the genus $2 n$ region. Because of the symmetry of the ZS system, $\mathcal{R}(x, \tau)$ is Schwarz-symmetrical. The surface $\mathcal{R}(x, \tau)$ and, more precisely, its $2 n+1$ complex branch points (because of the symmetry, we consider only branch points in the upper half-plane), do not depend on the semiclassical parameter $\varepsilon$. They can be viewed as slowly varying functions of the space-time variables that describe the wave's parameters, i.e., the macroscopic structure of the solution in the vicinity of a given point $x, \tau$.

Equations that define the branch points of $\mathcal{R}(x, \tau)$ are known as modulation or Whitham equations. In the case $n=0$ (genus zero case), $\mathcal{R}(x, \tau)$ has only two branch points: $\alpha=\alpha(x, \tau)$ and its complex conjugate $\bar{\alpha}$. In this case, the Riemann Theta-function expression for $q(x, \tau, \varepsilon)$ is replaced by

$$
q(x, \tau, \varepsilon)=A(x, \tau) e^{i S(x, \tau) / \varepsilon}+O(\varepsilon),
$$

where

$$
\alpha(x, \tau)=-\frac{1}{2} S_{x}(x, \tau)+i A(x, \tau),
$$

and $A(x, 0)=A(x), S(x, 0)=S(x)$. The genus zero region is the first region adjacent to the $\tau=0$ axis where the solution (16) has the form of a high frequency $\mathcal{O}(1 / \varepsilon)$ modulated wave with slowly varying amplitude $A(x, \tau)$ and phase $S(x, \tau)$.

For the initial data (14), the corresponding scattering data for (8) was calculated explicitly [23] (For general initial data of the type (15) see Sec. V). Using this data, the modulated amplitude and phase of (16) and (17) can be obtained from the system of transcendental equations for $\alpha(x, \tau)=a(x, \tau)+i b(x, \tau)$ :

$$
\left\{\begin{array}{l}
\sqrt{(a-T)^{2}+b^{2}}+\sqrt{(a+T)^{2}+b^{2}}=\mu+2 \tau b^{2}, \\
{\left[a-T+\sqrt{(a-T)^{2}+b^{2}}\right]\left[a+T+\sqrt{(a+T)^{2}+b^{2}}\right]} \\
=b^{2} e^{2(x+2 \tau a)}
\end{array}\right.
$$

where $T=\sqrt{\frac{\mu^{2}}{4}-1}$. It is interesting to mention here that, in general, the phase gradient $S^{\prime}(x)$ has a significant impact on the asymptotic behavior of the evolving solution. For example [23], in the case $0 \leq \mu<2$, the 
corresponding ZS eigenvalue problem has $O(1 / \varepsilon)$ points in the discrete spectrum (solitons) located on the vertical segment $[-T, T]$. These solitons are localized at $x=0$, do not move, and their interaction can produce quite complicated coherent structures in the process of evolution. In Fig. 1, these structures are seen after the second break (around $x=0$ and $\tau>1.5$ ), which, presumably, corresponds to the genus 4 region. It is expected that the solution undergoes more phase transitions (breaks) for larger values of time $\tau$ that are not visible in Fig. 1. On the contrary, in the case $\mu>2$, the ZS eigenvalue problem does not have any discrete spectrum (solitons). The evolution of such initial data produces only two asymptotic regimes (of genera zero and two), similar to the first two asymptotic regions in Fig. 1 (the second asymptotic region extends to $t=\infty$, see [12] for the proof). The breaking curve separating these two regions has linear (slanted) asymptotes as $\tau \rightarrow \infty$. In the limit $\tau \rightarrow \infty$, the solution in the genus zero region approaches zero exponentially fast, whereas in the genus two region (inside the wedge) it decays as $O\left(\tau^{-\frac{1}{2}}\right)$ [12]. The high frequency amplitude oscillations in this region decay exponentially in $\tau$, and the solution has the profile of a parabola with a maximum at $x=0$ and zero values along the breaking curve. Qualitatively, these results mean that a large focusing momentum (directed towards the origin), generated by the phase gradient $S^{\prime}(x)=-\mu \tanh x$ with $\mu>2$, prevents the formation of the higher genera regions.

In the borderline case $\mu=2$, equations (18) have a particularly simple solution. Introducing the implicit time $u=u(x, \tau)$ at each point $x \in \mathbb{R}$ by $\tau=(u-x)[\sinh 2 u-$ $(u-x)] /\left(4 \sinh ^{2} u\right)$, one can obtain an explicit solution of eq. (18) (see [12])

$$
a=\frac{2 \sinh ^{2} u}{\sinh 2 u-(u-x)}, \quad b=\frac{2 \sinh u}{\sinh 2 u-(u-x)}
$$

for $A(x, \tau)=b$ and $S^{\prime}(x, \tau)=-2 a$ that are valid throughout the genus zero region. Similar expressions with the implicit time $u=u(x, \tau)$ given by $\tau=$ $\frac{1}{2} \sqrt{(u-x)[\sinh 2 u-(u-x)]} \operatorname{coth} u$ and

$$
a^{2}=\frac{(u-x) \tanh ^{2} u}{\sinh 2 u-(u-x)}, \quad b^{2}=\frac{2 \tanh u}{\sinh 2 u-(u-x)}
$$

are valid in the case $\mu=0$.

Notice that the amplitude $A(x, t)$ of the solution in Fig. 1 at first contracts (focuses) towards the point of maximum amplitude, $x=0$, and then suddenly bursts into rapid (order $1 / \varepsilon$ ) and violent oscillations, transitioning to the genus two regime. This is typical behavior [24] for an analytic one-hump initial condition provided that $S^{\prime}(x)$ does not decrease too fast. The very first point of this transition, which is the tip-point of the first breaking curve (see Fig. 1), is called a point of gradient catastrophe [34]. At the point $\left(x_{0}, \tau_{0}\right)$ of the gradient catastrophe, the semiclassical solution (16) of (8) loses its smoothness [27], i.e., $\alpha_{x}\left(x_{0}, \tau_{0}\right)=\infty$ (either $A_{x}\left(x, \tau_{0}\right)$ or $S_{x x}\left(x, \tau_{0}\right)$ or both become infinite). The recent results of [38] show that each of the spikes seen in Fig. 1 immediately after the moment of gradient catastrophe represents a rational breather (see [35]) solution to the NLS. The height of each spike is exactly three times the value of the amplitude at the time of gradient catastrophe, i.e., $3 A\left(x_{0}, \tau_{0}\right)+O\left(\varepsilon^{1 / 5}\right)$ and the location of the spikes are determined by the poles of the tritronquée solution of the first Painlevé equation.

Due to the symmetry of the initial data (14), the gradient catastrophe occurs at $x_{0}=0$. In the cases $\mu=2$ and $\mu=0$, the time of the gradient catastrophe $\tau_{0}$ can be calculated as $\tau_{0}=\lim _{u \rightarrow 0} \tau(u, 0)$, where the expressions for $\tau=\tau(u, x)$ are given above formulae (19) and (20) respectively. This yields $\tau_{0}=1 / 4$ for $\mu=2$ and $\tau_{0}=1 / 2$ for $\mu=0$. The value of the amplitude $b_{0}=A\left(x_{0}, \tau_{0}\right)$ at the point of gradient catastrophe can be calculated as $b_{0}=\lim _{u \rightarrow 0} b(u, 0)$, where $b=b(u, x)$ are given in (19) and in (20). Thus, $b_{0}=2$ for $\mu=2$ and $b_{0}=\sqrt{2}$ for $\mu=0$. In the case $\mu=0$, numerical solution of (8) with $\varepsilon=1 / 33$ is shown on Fig. 2 .

One possible mechanism for the loss of onedimensionality described by our 1D analysis is the generation of large axial momentum. This may correspond to the breaking down of the energetic assumption of weak axial kinetic energy relative to the radial harmonic oscillator energy (see eqs. (6) and (A.3)). To illustrate this point, we consider the evolution of the Fourier transform

$$
\hat{q}(p, \tau, \varepsilon)=\frac{1}{\sqrt{2 \pi}} \int_{\mathbb{R}} A(x, \tau) e^{\frac{i}{\varepsilon}[S(x, \tau)+\varepsilon p x]} d x
$$

of the solution $q(x, \tau, \varepsilon)$ to (8) in the genus zero region (where $q(x, \tau, \varepsilon)$ has the form (16)). If we define the relative momentum $P$ through the standard momentum $p$ as $P=\varepsilon p$, the standard stationary phase method can be applied to (21). Time evolution of $\frac{1}{\sqrt{\varepsilon}}|\hat{q}(P / \varepsilon, \tau, \varepsilon)|$ that corresponds to the initial data (15) with $A(x)=\operatorname{sech} x$ and $S^{\prime}(x)=-2 \tanh x$ is shown on Fig. 3 (in this case $A(x, \tau)$ and $S(x, \tau)$ are given by (17) and (19)). In fact, direct calculations show that in the leading $\varepsilon$-order $\frac{1}{\sqrt{\varepsilon}}|\hat{q}(P / \varepsilon, 0, \varepsilon)|=\frac{1}{\sqrt{2}}$ and $\frac{1}{\sqrt{\varepsilon}}|\hat{q}(P / \varepsilon, 1 / 4, \varepsilon)|=\sqrt{|P| / 2}$ when $|P| \leq 2$ and $\frac{1}{\sqrt{\varepsilon}} \hat{q}(P / \varepsilon, \tau, \varepsilon)=0$ when $|P|>2$ and $0 \leq \tau \leq 1 / 4$. Explicit leading order expressions can be obtained for any $0 \leq \tau \leq 1 / 4$. They show that the portion of atoms in the condensate with high axial momentum significantly increases (see Fig. 3) as the point of gradient catastrophe at $\tau=0.25$ is approached.

\section{CALCULATION OF SEMICLASSICAL NLS SOLUTIONS FOR GENERAL ONE-HUMP INITIAL DATA}

Equation (8), the integrable NLS, can be solved by the inverse scattering technique. However, the semiclassical limit solutions require the semiclassical limit of the scattering transform. Let $\Sigma$ be the curve in the upper half plane, defined parametrically by the analytic initial data $(15)$ as $\alpha(x)=-\frac{1}{2} S^{\prime}(x)+i A(x), x \in \mathbb{R}$. Here $A(x)$ and 


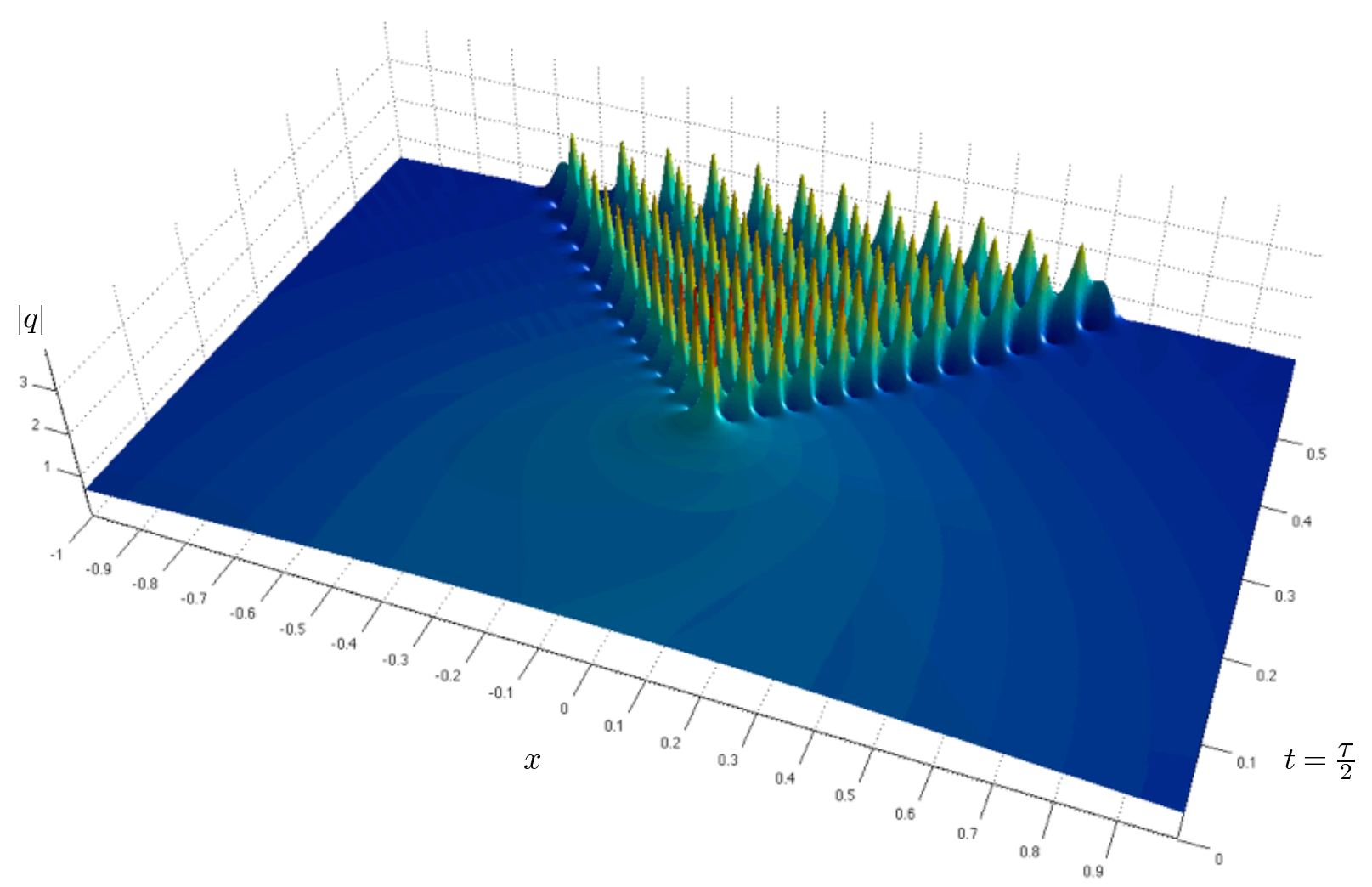

FIG. 2: Numerical simulation of the solution of (8) with the initial data (14), where $\mu=0, \varepsilon=1 / 33$. The time scale $t$ used here and our time $\tau$ are related through $\tau=2 t$. This simulation confirm the values $\tau_{0}=0.5$ and $b_{0}=\sqrt{2}$. It also shows that each spike near the point of gradient catastrophe has the height of $3 b_{0}$ (with the accuracy $\left.(1 / 33)^{1 / 5} \approx 0.496\right)$ and the shape of a rational breather.

$S^{\prime}(x)-\mu_{ \pm}$, where $\mu_{ \pm}$are some real numbers, have sufficient decay as $x \rightarrow \pm \infty$ respectively. Let $z$ be a point on $\Sigma$. Assuming for simplicity that $\alpha(x)$ is invertible, the semiclassical scattering data limit $f_{0}(z), z \in \Sigma$, is defined $[27]$ through the generalized Abel integral transform as

$$
\begin{aligned}
f_{0}(z)=\int_{z}^{\mu_{+}} & {\left[z-\mu_{+}+\sqrt{(z-u)(z-\bar{u})}\right] x^{\prime}(u) d u } \\
& +\left(z-\mu_{+}\right) x(z),
\end{aligned}
$$

where $x(\alpha)$ is inverse to $\alpha(x)$ and the integral is taken along $\Sigma$. The analytic extension of $f_{0}(z)$ from $\Sigma$ to $\mathbb{R}$ (which can have logarithmic branch cuts) has a meaning of the leading order term of $\frac{1}{2} i \varepsilon \ln r_{0}(z, \varepsilon)$ as $\varepsilon \rightarrow 0$, where $r_{0}(z, \varepsilon), z \in \mathbb{R}$, is the reflection coefficient of (15). Once $f_{0}(z)$ is known, the complex wave parameters encoded in the branch points of $\mathcal{R}(x, \tau)$ are defined through the modulation equations. In particular, in the genus zero region, the modulation equation for $\alpha(x, \tau) \in \mathbb{C}^{+}$is given by the system of two real equations [12]

$$
\int_{\gamma} \frac{f^{\prime}(\zeta)}{R(\zeta)} d \zeta=0, \quad \int_{\gamma} \frac{\zeta f^{\prime}(\zeta)}{R(\zeta)} d \zeta=0
$$

where $f(z)=f(z ; x, \tau)=f_{0}(z)-x z-\tau z^{2}$, $R(z)=\sqrt{(z-\alpha)(z-\bar{\alpha})}$ and $\gamma$ is an oriented, Schwarz- symmetrical contour connecting $\bar{\alpha}$ and $\alpha$, such that $\gamma \cup \mathbb{R}=\mu_{+}$. It defines $q(x, \tau, \varepsilon)$ through (16)-(17). Here $f_{0}(z)$ is Schwarz symmetrically extended into the lower half plane; typically $\Im f_{0}(z)$ has a jump along $\mathbb{R}$.

We define the function $h(z)=h(z ; x, \tau)$ as

$$
h(z)=\frac{R(z)}{i \pi} \int_{\gamma} \frac{f(\zeta)}{(\zeta-z) R(\zeta)} d \zeta-f(z) .
$$

Because of the analyticity of $f(z)$, the particular shape of $\gamma$ is not important. However, it is possible to fix $\gamma$ by the condition $\Im h(z)=0$ on $\gamma$. According to the Deift-Zhou nonlinear steepest descent method [36], the genus zero ansatz (16) approximates the actual solution of the NLS (8) with the reflection coefficient $r_{0}(z, \varepsilon)=e^{-\frac{2 i}{\varepsilon} f_{0}(z)}$ if (see [12]) simultaneously:

$$
\begin{aligned}
& \Im h(z ; x, \tau)<0 \text { on both sides of } \gamma^{+} ; \\
& \Im h(z ; x, \tau)>0 \text { on } \gamma_{c}^{+},
\end{aligned}
$$

where $\gamma_{c}^{+}$is a contour in the upper half plane $\mathbb{C}^{+}$connecting $\alpha$ and $\mu_{-}$and $\gamma^{+}=\gamma \cap \mathbb{C}^{+}$. We have the freedom to deform the contours $\gamma, \gamma_{c}^{+}$so that the inequalities (25) are satisfied along it. The first breaking curve consists of points $(x, \tau)$ where at least one of the inequalities $(25)$ 


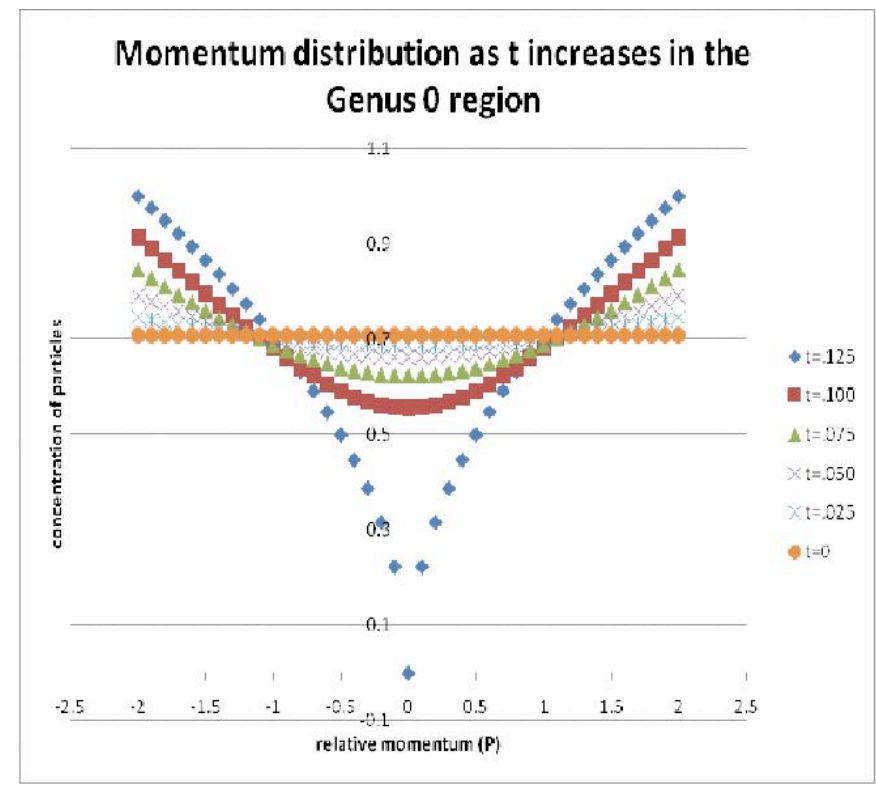

FIG. 3: Evolution of the Fourier transform of the initial data (15) with $A(x)=\operatorname{sech} x$ and $S^{\prime}(x)=-2 \tanh x$ in the limit $\varepsilon \rightarrow 0$ from $\tau=0$ to the time of gradient catastrophe $\tau=0.25$; time $t$ shown on the figure and $\tau$ are related by $\tau=2 t$. The vertical axis shows the values of $\frac{1}{\sqrt{\varepsilon}}|\hat{q}|$, the horizontal axis shows the relative momentum $P$, which is defined as $P=$ $\varepsilon p$, where $p$ is the standard momentum. The data shown here is explicitly calculated from (21) through the standard stationary point method.

turns into equality at some $z_{0}$. Thus, the equation for the first breaking curve can be written as a system of three real equations for $z_{0} \in \mathbb{C}$ and $(x, \tau) \in \mathbb{R}^{2}$

$\Im h\left(z_{0} ; x, \tau\right)=0 \quad$ and $\quad h_{z}\left(z_{0} ; x, \tau\right)=0$.

For the initial data (14) with $\mu=2$, the expression

$$
\begin{aligned}
h(z)= & z \ln \frac{\sqrt{a^{2}+b^{2}} R(z)-a(z-a)+b^{2}}{z}- \\
& \tau(z-a) R(z)+(1-z)\left[\ln b-\frac{i \pi}{2}\right]- \\
& \ln [R(z)-(z-a)],
\end{aligned}
$$

was found in [12]. The modulation equations, as well as expressions for $h(z ; x, \tau)$ for higher genus regions, can be written in explicit determinantal form (see $[25,26]$ ). Since these expressions are somewhat involved, they will not be given in this paper.

The point of gradient catastrophe $x_{0}, \tau_{0}$ is defined by the system of equations that consists of (23) and the following two equations (see [24]):

$$
\int_{\gamma} \frac{f^{\prime \prime}(\zeta)}{R_{+}(\zeta)} d \zeta=0, \quad \int_{\gamma} \frac{\zeta f^{\prime \prime}(\zeta)}{R_{+}(\zeta)} d \zeta=0 .
$$

This is a system of four real equations for $\alpha \in \mathbb{C}$ and $\left(x_{0}, \tau_{0}\right) \in \mathbb{R}^{2}$.
Equations (22)-(28) show that the $\mathcal{O}(\varepsilon)$-approximate solution in the genus zero region, the point of gradient catastrophe, and the breaking curve can be effectively calculated from decaying initial data of the form (15). Further calculations also reveal the asymptotic structure of the solution in the genus two region near the first breaking curve and around the point of gradient catastrophe, see [37] and [38] respectively.

\section{SUGGESTIONS AND CONCLUSIONS}

The semiclassical limit of the focusing 1D NLS (8) provides a new, mathematically rigorous tool to study the modulationally unstable evolution of an attractive 1D BEC. When the conditions $N<N_{\text {cr }}$ (see eq. (5)) and inequalities (6), (10)-or eq. (12)-are satisfied, an attractive BEC in an extended cylindrical trap (cigar shaped potential without axial caps) is expected to be governed by the focusing NLS in the semiclassical regime. A typical evolution for decaying, single-hump, analytic initial data $q(x, 0, \varepsilon)$, see $(15)$, is depicted in Fig. 1. Using new tools from asymptotic inverse scattering theory, in particular, the Deift-Zhou nonlinear steepest descent method, outlined above, we were able to calculate the solution with $\mathcal{O}(\varepsilon)$ accuracy in several different regimes. We have obtained a number of macroscopic characteristics of the evolving condensate. These include (see Fig. 1) the space-time location of the point of gradient catastrophe and the breaking curves, the slowly modulated amplitude in the genus zero region, and the envelope of the fast amplitude oscillations in the genus two region.

Attractive 1D BEC experiments without axial trap are expected to produce two counterpropagating radiative waves with the space in between filled with two-phase modulated waves and, possibly, with spatially localized coherent structures that consist of more complicated nonlinear waves. These higher genus stationary structures are expected to be linked with the discrete spectrum of the corresponding $\mathrm{ZS}$ system.

The calculation of the observables, mentioned above, is based on the semiclassical limit of the scattering data $f_{0}(z)$, which, in its turn, can be obtained from $q(x, 0, \varepsilon)$ through (22), i.e., through the initial amplitude $A(x)$ and the phase $S(x)$. However, accurate measurement of the initial phase is often a difficult task. We can turn the question around and ask whether the phase $S(x)$ can be somehow reconstructed from $A(x)$ and some observables. Continuation of this line of argument leads to the question of designing some NLS data, initial or scattering, whose evolution will have certain desired properties and/or fit within some required parameters. In light of the described above example of the initial amplitude $A(x)=\operatorname{sech} x$ and the phase gradient $S^{\prime}(x)=-\mu \tanh x$, it seems to be especially interesting to observe experimentally how increasing the phase gradient $S^{\prime}(x)$ for the same amplitude $A(x)$ (by, for example, applying an external focusing potential) can reduce the number of phase 
transitions of the evolving condensate.

Generally speaking, formulae (22)-(26) are valid for a large class of analytic initial data, including, for example, multi-hump initial data. Further theoretical and experimental investigations of multi-hump initial densities may yield interesting results.

The authors thank V. Kokoouline for stimulating discussions and B. Relethford, who participated in the summer 2009 REU DMS 0649159 at the UCF under the supervision of the first author, for calculating the Fourier transform $\hat{q}(p, \tau, \varepsilon)$ and creating Figure 3 .

\section{Appendix: Some energy estimates}

In this appendix, we briefly outline the derivation of the quasi-1D criterion (6).

The conserved energy associated with the 3D GP equation (1) for $t>0$ (when $V(\mathbf{r}, t)=V_{\perp}(y, z)$ and $g(t)=$ $\left.4 \pi \hbar^{2} a_{s}^{(a)} / m<0\right)$ is

$$
\begin{aligned}
\mathcal{E}[\Psi]= & \mathcal{E}_{\perp}+\mathcal{E}_{\|}+\mathcal{E}_{\mathrm{nl}} \\
\equiv & \int_{\mathbb{R}^{3}}\left\{\frac{\hbar^{2}}{2 m}\left|\nabla_{\perp}^{\prime} \Psi\right|^{2}+V_{\perp}\left(y^{\prime}, z^{\prime}\right)|\Psi|^{2}\right\} d \mathbf{r}^{\prime}+ \\
& \int_{\mathbb{R}^{3}} \frac{\hbar^{2}}{2 m}\left|\Psi_{\xi^{\prime}}\right|^{2} d \mathbf{r}^{\prime}+\int_{\mathbb{R}^{3}} \frac{g}{2}|\Psi|^{4} d \mathbf{r}^{\prime} .
\end{aligned}
$$

Assuming that $\Psi$ has the approximate separated form given in eq. (7), we formally compute

$$
\begin{aligned}
\mathcal{E}_{\perp} & =\frac{N \omega_{\perp} \hbar}{2 \pi}\|q(\cdot, \tau)\|_{L^{2}(\mathbb{R})}^{2}, \\
\mathcal{E}_{\|} & =\frac{\hbar^{2} N}{4 \pi m \Delta^{2}}\left\|q_{z}(\cdot, \tau)\right\|_{L^{2}(\mathbb{R})}^{2}, \\
\mathcal{E}_{\mathrm{nl}} & =\frac{\hbar a_{s}^{(a)} N^{2} \omega_{\perp}}{2 \pi \Delta}\|q(\cdot, \tau)\|_{L^{4}(\mathbb{R})}^{4},
\end{aligned}
$$

where $\|f(\cdot)\|_{L^{p}(\mathbb{R})} \equiv\left(\int_{\mathbb{R}}|f(x)|^{p}\right)^{1 / p}$ is the standard $L^{p}$ norm. If the characteristic energies of the radial harmonic oscillator excitations $\delta \mathcal{E}_{\perp} \geq \mathcal{E}_{\perp}$ are much greater then the energies associated with the axial $\mathcal{E}_{\|}$and nonlinear $\mathcal{E}_{\mathrm{nl}}$ excitations, the 3D GP equation (1) can be approximated by a 1D GP equation in the longitudinal (axial) direction [21, 22]. For the separated ansatz (14) and the 1D NLS equation (8) to be valid, we therefore assume

$$
\mathcal{E}_{\perp} \gg \mathcal{E}_{\|}, \quad \mathcal{E}_{\perp} \gg\left|\mathcal{E}_{\mathrm{nl}}\right|
$$

or, since the $L^{2}$ norm of $q$ is unity (see eq. (11)),

$$
\frac{a_{0}^{2}}{2 \Delta^{2}}\left\|q_{z}(\cdot, \tau)\right\|_{L^{2}(\mathbb{R})}^{2} \ll 1, \quad \frac{\left|a_{s}^{(a)}\right| N}{\Delta}\|q(\cdot, \tau)\|_{L^{4}(\mathbb{R})}^{4} \ll 1
$$

Further simplification can be made by using the fact that the 1D energy for $q$ satisfying the NLS equation (8) is conserved

$$
\mathcal{E}_{1 \mathrm{D}}=\frac{\varepsilon^{2}}{2}\left\|q_{z}(\cdot, \tau)\right\|_{L^{2}(\mathbb{R})}^{2}-\frac{1}{2}\|q(\cdot, \tau)\|_{L^{4}(\mathbb{R})}^{4} .
$$

For slowly varying, single-hump (Gaussian type) initial data in the semiclassical regime (10) satisfying (11), we will have $\mathcal{E}_{1 \mathrm{D}}<0$. Then the inequalities in (A.3) become

$$
\begin{aligned}
& \frac{N\left|a_{s}^{(a)}\right|}{\Delta}\left(\|q(\cdot, \tau)\|_{L^{4}(\mathbb{R})}^{4}-2\left|\mathcal{E}_{1 \mathrm{D}}\right|\right) \ll 1, \\
& \frac{N\left|a_{s}^{(a)}\right|}{\Delta}\|q(\cdot, \tau)\|_{L^{4}(\mathbb{R})}^{4} \ll 1 .
\end{aligned}
$$

If we have

$$
\max _{x \in \mathbb{R}}\left(|q(x, \tau)|^{2}\right) \leq C, \quad \tau \in\left[0, T_{0}\right]
$$

then the $L^{4}$ norm of $q$ is bounded by $C$ because

$$
\|q(\cdot, \tau)\|_{L^{4}(\mathbb{R})}^{4} \leq \max _{x \in \mathbb{R}}\left(|q(x, \tau)|^{2}\right)\|q(\cdot, \tau)\|_{L^{2}(\mathbb{R})}^{2} \leq C
$$

for $\tau \in\left[0, T_{0}\right]$ by use of eq. (11). Then the inequalities in (A.6) leading to a quasi-1D BEC are satisfied when eq. (6) holds. Using (11) and our semiclassical calculations, we can estimate $C \approx 9\left|q\left(x_{0}, \tau_{0}\right)\right|^{2}$ for $\tau \in\left[0, T_{0}\right]$ with $T_{0}>\tau_{0}$ but $T_{0}<\tau_{1}$, the time of the second break. For example, the initial data in eq. (14) give $C \lesssim 9(\mu+2)$.
[1] J.M. Gerton, D. Strekalov, I. Prodan, and R. G. Hulet, Nature 408, 692-695 (2000).

[2] E.A. Donley, N.R. Claussen, S.L. Cornish, J.L. Roberts, E.A. Cornell, and C.E. Wieman, Nature 412, 295-299 (2001).

[3] S.L. Cornish, S.T. Thompson, and C.E. Wieman, Phys. Rev. Lett., 96, 170401 (2006).

[4] K.E. Strecker, G.B. Partridge, A.G. Truscott, and R.G. Hulet, Nature 417, 150-153 (2002).

[5] L. Khaykovich, F. Schreck, G. Ferrari, T. Bourdel, J. Cubizolles, L.D. Carr, Y. Castin, and C. Salomon, Science 296, 1290-1293 (2002).
[6] P.A. Ruprecht, M.J. Holland, K. Burnett, and M. Edwards, Phys. Rev. A 51, 4704 (1995).

[7] T. Tsurumi and M. Wadati, J. Phys. Soc. Japan 68, 1531-1536 (1999).

[8] A. Gammal, T. Frederico, and L. Tomio, Phys. Rev. A, 64, 055602, (2001).

[9] P.G. Kevrekidis and D.J. Frantzeskakis, Mod. Phys. Lett. B, 18, 173-202 (2004).

[10] B.P. Anderson, P.C. Haljan, C.A. Regal, D.L. Feder, L.A. Collins, C.W. Clark, and E.A. Cornell, Phys. Rev. Lett., 86, 2926 (2001). Z. Dutton, M. Budde, C. Slowe, and L.V. Hau, Science, 293, 663 (2001). 
[11] S.E. Pollack, D. Dries, M. Junker, Y.P. Chen, T.A. Corcovilos, and R.G. Hulet, Phys. Rev. Lett. 102, 090402 (2009).

[12] Tovbis, A., Venakides, S. and Zhou, X., Comm. Pure Appl. Math., 57, 877-985, (2004).

[13] Kamvissis, S., McLaughlin, K. T.-R. and Miller, P., Ann. Math. Stud., 154, Princeton University Press, Princeton, (2003).

[14] Tovbis, A., Venakides, S. and Zhou, X., Comm. Pure and Appl. Math., 59, 1379-1432 (2006).

[15] C. J. Pethick and H. Smith, Bose-Einstein Condensation in Dilute Gases (Cambridge University Press, Cambridge, 2001).

[16] L. P. Pitaevskii and S. Stringari, Bose-Einstein condensation (Clarendon, Oxford, 2003).

[17] R. Carles, Ann. H. Poincare, 3, 757-772, (2002).

[18] L. Berge, T. J. Alexander, and Y. S. Kivshar, Phys. Rev. A, 62, 023607 (2000).

[19] H.A. Rose and M.I. Weinstein, Physica D, 30, 207-218, (1988).

[20] N. G. Parker, S. L. Cornish, C. S. Adams, and A. M. Martin, J. Phys. B, 40, 3127 (2007).

[21] F. Kh. Abdullaev, A. Gammal, A.M. Kamchatnov and L. Tomio, Int. J. of Mod. Phys. B, 19, 3415-3473 (2005).

[22] P. G. Kevrekidis, D. J. Frantzeskakis, and R. CarreteroGonzlez, Emergent nonlinear phenomena in BoseEinstein condensates: theory and experiment (Springer, Berlin, 2008).

[23] Tovbis, A. and Venakides, S., Phys. D, 146 150-164 (2000).

[24] Tovbis, A., Venakides, S. and Zhou, X., IMRN 2254 (2007).
[25] Tovbis, A. and Venakides, S., arXiv:0803.2066 (2008).

[26] Tovbis, A. and Venakides, S., IMRN, 2056-2080, (2009).

[27] Tovbis, A. and Venakides, S., arXiv:0903.2648 (2009).

[28] Cai, D., McLaughlin, D.W. and McLaughlin, K.T.R., Handbook of dynamical systems, 2 599-675 (NorthHolland, Amsterdam, 2002).

[29] Zakharov, V. E. and Shabat, A. B., Sov. Phys. JETP, 34 62-69 (1972).

[30] Forest, M.G. and Lee, J.E., Oscillation Theory, Computation, and Methods of Compensated Compactness, C. Dafermos, et. al. (Eds.), 2 (Springer, New York, 1986).

[31] A. M. Kamchatnov, R. A. Kraenkel, and B. A. Umarov, Phys. Rev. E 66, 036609 (2002).

[32] Miller P. D. and Kamvissis, S., Phys. Lett. A, 247, 75-86 (1998).

[33] Ceniceros, H. and Tian, F.-R., Phys. Lett. A, 306, 25-34 (2002).

[34] Dubrovin, B., Grava, T., and Klein, C., J. Nonlin. Sci., 19, 57-94 (2009).

[35] N. Karjanto and E. van Groesen, Proc. 5th SEAMSGMU Int. Conf. Math. Appl, Yogyakarta, Indonesia (2007).

[36] Deift, P., Venakides, S. and Zhou, X., Internat. Math. Res. Notices, 6 286-299, (1997).

[37] M. Bertola and A. Tovbis, Universality in the profile of the nonlinear Schrödinger equation at the first breaking curve, Int. Math. Res. Not., (2009) rnp196, 49pp.

[38] M. Bertola and A. Tovbis, Universality for the focusing Nonlinear Schrödinger equation at gradient catastrophe point, in preparation. 